\title{
Correlation of measurable serum markers of inflammation with lung levels following bilateral femur fracture in a rat model
}

This article was published in the following Dove Press journal:

Journal of Inflammation Research

23 August 2010

Number of times this article has been viewed

\author{
Benjamin W Sears' \\ Dustin Volkmer' \\ Sherri Yong ${ }^{2}$ \\ Ryan D Himes' \\ Kristen Lauing' \\ Michele Morgan' \\ Michael D Stover' \\ John J Callaci' \\ 'Department of Orthopaedics, \\ 2Department of Pathology, Loyola \\ University Medical Center, Maywood, \\ IL, USA
}

Introduction: Evaluation of the systemic inflammatory status following major orthopedic trauma has become an important adjunct in basing post-injury clinical decisions. In the present study, we examined the correlation of serum and lung inflammatory marker levels following bilateral femur fracture.

Materials and methods: 45 Sprague Dawley rats underwent sham operation or bilateral femoral intramedullary pinning and mid-diaphyseal closed fracture via blunt guillotine. Animals were euthanized at specific time points after injury. Serum and lung tissue were collected, and 24 inflammatory markers were analyzed by immunoassay. Lung histology was evaluated by a blinded pathologist.

Results: Bilateral femur fracture significantly increased serum markers of inflammation including interleukin (IL)-2, IL-6, IL-10, GM-CSF, KC/GRO, MCP-1, and WBC. Femur fracture significantly increased serum and lung levels of IL-1a and $\mathrm{KC} / \mathrm{GRO}$ at 6 hours. Lung levels of IL-6 demonstrated a trend towards significance. Histologic changes in pulmonary tissue after fracture included pulmonary edema and bone elements including cellular hematopoietic cells, bone fragments and marrow emboli.

Discussion and conclusion: Our results indicate that bilateral femur fracture with fixation in rats results in increases in serum markers of inflammation. Among the inflammatory markers measured, rise in the serum KC/GRO (CINC-1), a homolog to human IL-8, correlated with elevated levels of lung KC/GRO. Ultimately, analysis of serum levels of KC/GRO (CINC-1), or human IL-8, may be a useful adjunct to guide clinical decisions regarding surgical timing.

Keywords: blunt trauma, injury, cytokine, IL-8, bone marrow emboli

\section{Introduction}

The magnitude of inflammatory response following major trauma has been implicated in the development of systemic complications including multiorgan failure and adult respiratory distress syndrome (ARDS). ${ }^{1}$ Along with the stress response from the initial traumatic insult, operative procedures have also been shown to contribute to the overall degree of the inflammatory response. ${ }^{2,3}$ Surgical stress is associated with the release of proinflammatory mediators, the priming of circulating neutrophils, and alterations in the fibrinolytic and coagulatory cascades, independent of the initial traumatic injury. ${ }^{1}$ An ill-timed or extensive surgical procedure in a multiply injured patient may propagate an already elevated inflammatory response placing the patient at risk for end-organ injury. Quantification of the inflammatory response in patients after major injury is important to help guide clinical decisions and optimize surgical timing.
Correspondence: Benjamin W Sears Loyola University Medical Center, Department of Orthopaedics, $2160 \mathrm{~S}$ Ist Ave, Maywood, IL 60I53, USA

Tel +l 7735909647

Fax+l 7082165858

Email bsears@lumc.edu 
However, assessment of the real-time inflammatory status in the post-trauma patient has proven difficult. This is because traditionally used clinical parameters, including urinary output, blood gases, ventilation status, and vital signs have limited sensitivity in screening patients at risk for inflammatory injury. ${ }^{4}$ Because of the concern for pulmonary injury in this population, spelcifically the development of post-fracture fixation ARDS, screening for elevated levels of inflammation may be beneficial in optimizing timing for surgical intervention. Currently the diagnosis of ARDS is based on clinical-radiological parameters, which develop after the onset of disease. ${ }^{5}$ Bronchoalveolar lavage and lung biopsy can be utilized for the evaluation of pulmonary injury; however, these tests are invasive and are not useful as a screening tool for most trauma patients in the acute postinjury setting. Identifying a readily available serum marker or panel that would assist in the assessment of progression of inflammation or pulmonary inflammatory risk would be a useful clinical tool.

The use of serum inflammatory markers in the evaluation of the inflammatory response has been shown to be clinically useful, readily accessible and may improve sensitivity in prediction of potential for injury. ${ }^{6,7}$ Advancing laboratory technology has improved the ability to analyze specific markers of inflammation, and some investigations have evaluated the utility of analyzing inflammatory markers in polytrauma patients. ${ }^{69} \mathrm{~A}$ direct relationship has been established between elevated levels of IL-6 and IL-8 and degree of injury. ${ }^{7}$ IL-6 levels have also been shown to be a reliable marker of the magnitude of systemic inflammation, ${ }^{10,11}$ and early post-injury elevation in IL-6 levels has been shown to accurately differentiate trauma patients who would later develop organ failure. ${ }^{12}$ As the biologic basis of the inflammatory response is better understood, the use of clinically measurable inflammatory markers will improve the perioperative analysis of multiply injured patients.

Several studies have demonstrated that traumatic femur fracture in rodents initiates inflammatory-related pathologic changes in lung tissue, including lung weight changes (pulmonary edema), increases in concentration of exhaled nitric oxide, increased protein in bronchoalveolar lavage, pulmonary hypertension, increases in capillary filtration coefficient, and increases in myeloperoxidase levels. ${ }^{13,14}$ However, no investigations have demonstrated a relationship of serum inflammatory markers with lung markers following significant orthopedic injury. Correlating easily measurable serum inflammatory markers with pulmonary markers would be a useful tool to assist in assessing lung tissue for inflammatory damage in the timing of surgical intervention following major orthopedic injury. We hypothesize that an increase in serum markers of inflammation will occur following bilateral femur fracture with stabilization in a rat model, and serum increases will also correlate with changes in markers of pulmonary inflammatory response.

\section{Methods}

\section{Experimental design}

The Loyola University Institutional Animal Care and Use Committee (IACUC) approved the animal protocol used in this investigation. 45 male Sprague Dawley rats (average weight, $400 \mathrm{~g}$ ) were used in these experiments. Animals were subjected to either sham operation (Sham, n-9) consisting of induction of anesthesia and sacrifice, or bilateral femoral intramedullary pinning followed by mid-diaphyseal closed fracture. Animals were euthanized at 4 specific time periods: at 6 hours (n-9), 24 hours (n-9), 48 hours (n-9), and 72 hours after injury (n-9). Rats were rendered unconscious by $\mathrm{CO}_{2}$ inhalation and killed by decapitation. At time of sacrifice, fracture creation was confirmed via dissection, and serum and lung tissue was collected.

\section{Fracture creation and animal care}

The rats were anesthetized with an intraperitoneal injection of ketamine $(90 \mathrm{mg} / \mathrm{kg})$ and xylazine $(10 \mathrm{mg} / \mathrm{kg})$. Before incision, the animals were administered pre-operative antibiotics to ensure aseptic surgery (gentamycin $5 \mathrm{mg} / \mathrm{kg}$ ) and buprinex $(0.15 \mathrm{mg} / \mathrm{kg})$ for post-operative pain in concordance with an established laboratory animal open surgical protocol. After the animal's hind limb was shaved and washed with a providoneiodine solution, a medial parapatellar incision (approximately $0.5 \mathrm{~cm}$ ) was created. With the knee extended, the patella was dislocated laterally and the medullary canal was entered through the intercondylar notch. A Dremel Moto-Tool drill (Mt. Prospect, IL) with a $1 \mathrm{~mm}$ drill bit was used to penetrate the articular cartilage and subchondral bone in order to enter the intramedullary canal. The canal was reamed with a 21-gauge needle, and a $1.25 \mathrm{~mm}$ Kirschner wire (Synthes, Monument, CA) was inserted through the medullary canal into the proximal part of the greater trochanter. The distal portion of the pin was cut flush with the femoral condyles so as not to interfere with knee function. The patella was reduced, and the extensor mechanism and skin were closed with 4-0 Vicryl resorbable sutures. After the incision was closed, the diaphysis of both pinned femurs was fractured with the use of a blunt guillotine 3-point bending apparatus as described previously. This device consisted of a $550 \mathrm{gm}$ weight dropped $15 \mathrm{~cm}$ driving a blunt 
guillotine down onto an outstretched rat leg placed across an open platform, creating a 3-point bending mechanism. ${ }^{15}$ This resulted in creating a closed femur fracture with soft tissue injury consistent with a blunt force injury (Figure 1). Following injury, animals were resuscitated with a $5 \mathrm{cc}$ IP injection of normal saline and were allowed to walk freely. Animals were caged in pairs, and food was placed on the floor of the cage, and the animals were able to drink freely from their water bottle. Animals surviving for more than 24 hours were administered buprinex for pain control and saline injection $(2 \mathrm{cc})$ for fluid resuscitation every 10-12 hours. No animals were sacrificed for pain control or abnormal behavior.

\section{Serum and lung cytokine assay}

Collected blood was immediately placed on ice and allowed to clot for 1 hour. Blood was then centrifuged ( $250 \mathrm{~g}$-force) for 10 minutes. Serum was collected, immediately placed into aliquots, and stored at $-80^{\circ} \mathrm{C}$ until analysis. Serum analysis of cytokines (Figure 2) was determined by ELISA kits (BD Bioscience, San Diego, CA), including IL-6, IL-10, TNF- $\alpha$, and CRP, or via Lincoplex assay service section (Millipore, St Charles, MO). All samples were run with a dilution of $1: 2$ in PBS with $2 \%$ bovine serum albumin. WBC differentials were performed using a HemaVet 950 FS Veterinary Hematology System (Drew Scientific, Dallas, TX). All samples were run in duplicates, and the average value is reported.

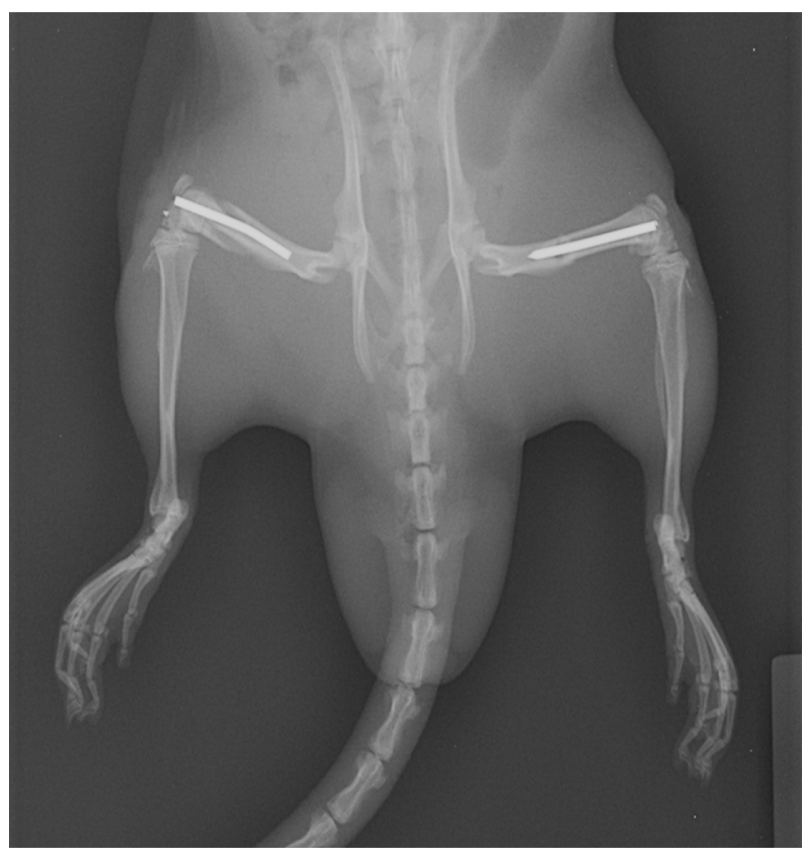

Figure I Surgical stabilization radiograph (General Electronic model Discovery $X R 650$, Fairfield, CT) following injury and euthanization, demonstrating typical femur fracture pattern caused by the blunt guillotine and appropriate placement of intramedullary fixation.

\begin{tabular}{|l|}
\hline $\begin{array}{l}\text { Inflammatory } \\
\text { Markers Investigated }\end{array}$ \\
\hline Eotaxin \\
\hline IL-1 $\alpha$ \\
\hline MCP-1 \\
\hline GM-CSF \\
\hline G-CSF \\
\hline Leptin \\
\hline MIP-1 $\alpha$ \\
\hline IL-4 \\
\hline IL-1 $\beta$ \\
\hline IL-2 \\
\hline IL-6 \\
\hline IL-9 \\
\hline IL-13 \\
\hline IL-10 \\
\hline IL-12p70 \\
\hline IL-5 \\
\hline IFN- $\gamma$ \\
\hline IL-17 \\
\hline IL-18 \\
\hline IP-10 \\
\hline GRO/KC \\
\hline RANTES \\
\hline TNF- $\alpha$ \\
\hline VEGF \\
\hline
\end{tabular}

Figure 224 inflammatory markers were investigated in serum and pulmonary tissue via immunoassay.

Lungs were collected immediately after euthanasia. A longitudinal midline thoracotomy incision was made, and the ribs separated from the sternum. The pulmonary vasculature was isolated and severed. The pulmonary tree was isolated, and the right upper lung lobe was separated and snap-frozen in liquid nitrogen and stored at $-80^{\circ} \mathrm{C}$ until processing. Lungs were prepared for ELISA analysis by adding $1 \mathrm{~mL}$ of buffer (Roche Mini Complete, EDTA free protease inhibitor cocktail) to an average of $140 \mathrm{mg}$ of sample. This solution was then subjected to a single freeze-thaw cycle at $-80^{\circ} \mathrm{C}$ and subsequent homogenizing, sonicating, and filtering prior to aliquot and refreeze until analysis at $-80^{\circ} \mathrm{C}$.

Lung analysis was also conducted by the Lincoplex assay service section (Millipore, St. Charles, MO). These samples were also run in duplicates and analyzed with BCA protein assay kit (Pierce Biotechnology, Rockford, IL). Again, the average value of the duplicates is reported. 


\section{Lung histology}

Lung tissue was harvested from animals immediately after sham procedure, at 6 hours and 24 hours following bilateral femur fracture and fixation. Lung tissue was fixed in $10 \%$ buffered formalin, processed for histological examination and embedded in paraffin. 4 micron sections were cut, stained with hematoxylin and eosin and reviewed by a single pathologist, blinded to sample type. Digital images were taken of representative areas using an Olympus DP12 digital camera microscope with an Olympus BX41 light and daylight filter (Leeds Precision Instruments, Minneapolis, MN). Leukocyte infiltration was evaluated and graded similar to previously published criteria. ${ }^{16}$ Briefly, leukocyte infiltration was assessed for parenchyma/alveolar regions after the entire lung section was examined and the most cellular area identified. The scoring system utilizing the most effected $400 \times$ field was as follows: $0=$ no extravascular leukocytes; $1 \leq 10$ leukocytes, $2=10-45$ leukocytes, and $3 \geq 45$ leukocytes. Peribronchial and perivascular leukocyte infiltration was graded similarly with a $0-3$ score. Small $(0.1 \mathrm{~mm}$ or less $)$ intermediate $(>0.1-0.5 \mathrm{~mm})$ and large $(>0.5 \mathrm{~mm})$ caliber bronchial and vessels were examined and separately graded. The presence of pulmonary edema was graded as $0=$ none, $1=$ focal, $2=$ multifocal, and $3=$ diffuse. Degenerative alveolar structures were present if distended alveolar spaces with degenerative/reactive pneuomocytes were identified. Fluid in alveolar spaces was also evaluated.

\section{Lung wet-to-dry ratio}

Lung wet-to-dry ratio was conducted by harvesting the right upper lung lobe and measuring lung weight immediately after tissue harvest. Lungs were placed into a $70^{\circ} \mathrm{C}$ incubator for 72 hours and then reweighed. The ratio of the wet-to-dry weight was obtained and data was analyzed.

\section{Statistical analyses}

Statistical analysis was performed using the software package Systat 11 (Systat Software, Inc., San Jose, CA). One-way ANOVA was performed using Tukey's post-hoc test. The results are considered as significant at $P<0.05$.

\section{Results}

\section{Analysis of serum inflammatory markers}

As illustrated by Figure 3, the effect of bilateral rat femur fracture with fixation demonstrated a significant change from baseline in serum levels of inflammatory markers. Compared with sham animals, fracture caused the significant increase in serum levels of IL-2, IL-6, IL-10, GM-CSF, KC/GRO, MCP-1, and WBC. Increase in levels of IL- 6 and $\mathrm{KC} / \mathrm{GRO}$ occurred at 6 hours post injury before returning to baseline at 24 hours. Elevation of serum IL-2 and GM-CSF were found at 24,48 , and 72 hours post injury. IL-10 and WBC levels were found to be significantly elevated at 24 hours post fracture. MCP-1 levels were elevated at 24 and 48 hours before returning to baseline at 72 hours.

\section{Analysis of pulmonary inflammatory markers}

In animals sustaining bilateral femur fracture with fixation, lung levels of the inflammatory markers KC/GRO and IL-1a were found to be significantly increased compared with sham injury animals at 6 hours after injury. Lung levels of IL-6 were associated with a trend towards increased lung levels at 24 and 72 hours post-injury (Figure 4). Figure 5 illustrates the difference between serum and lung levels of $\mathrm{KC} / \mathrm{GRO}$ and IL-6. Notably, a significant increase in levels of GRO/ $\mathrm{KC}$ in both serum and lung tissue occurred at 6 hours after injury.

\section{Histological analysis of pulmonary tissue}

Lung tissues from animals euthanized 6 hours post-injury were found to have significant microscopic changes (Figures 6A, 6B, and 6C). These changes included parenchymal and alveolar leukocyte infiltration, perivascular leukocyte infiltration, pulmonary edema and bone marrow elements including cellular hematopoietic cells, fat cells, and in some cases, bone fragments (Figure 6D). Animals euthanized at 24 hours also demonstrated pathologic changes, including parenchymal and alveolar leukocyte infiltration, peribronchial leukocyte infiltration, and reduced but present levels of pulmonary edema and bone marrow emboli compared with controls. Of note, animals sacrificed at 6 hours after injury demonstrated increased levels of perivascular leukocyte infiltration when compared with controls, a finding not seen in the 24-hour group. Conversely, the 24-hour group demonstrated increased levels of peribronchial leukocyte infiltration, which was not seen in the 6-hour group.

\section{Wet-to-dry analysis}

Wet-to-dry measurements on rat lungs were analyzed, and the 6-hour and 24-hour groups were compared with the sham group. These samples did not demonstrate any statistically significant difference in wet-to-dry ratio when compared with the sham group. 
A

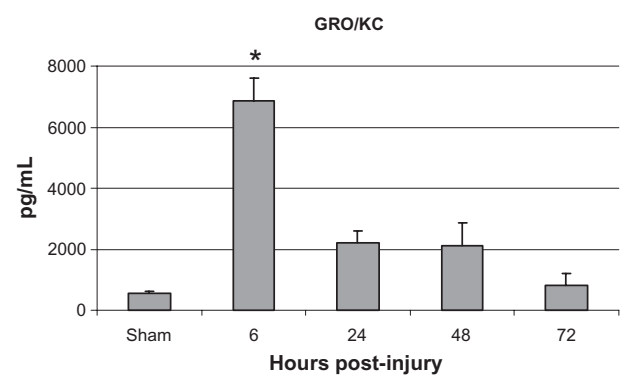

C

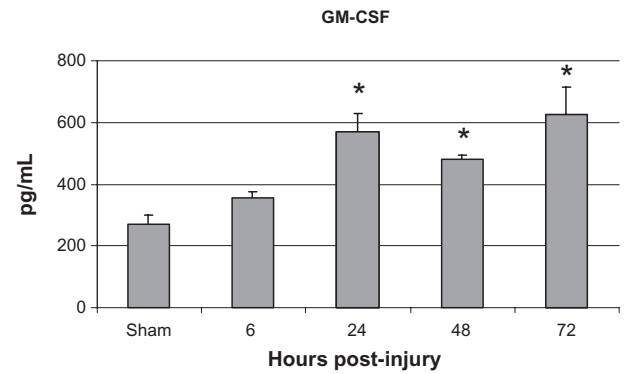

$\mathbf{E}$

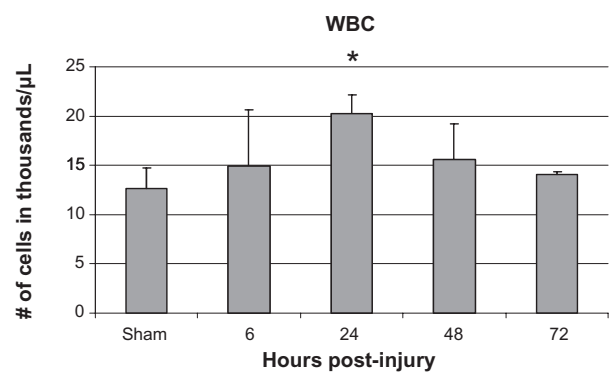

B

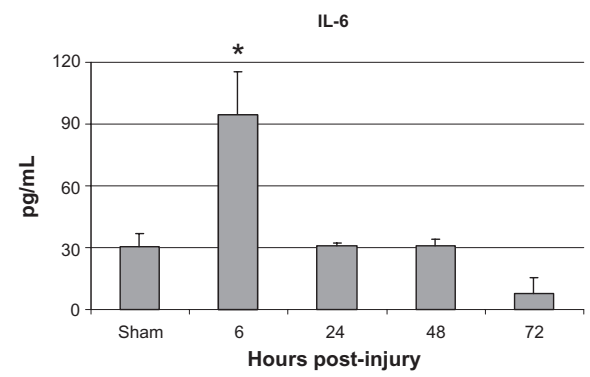

D

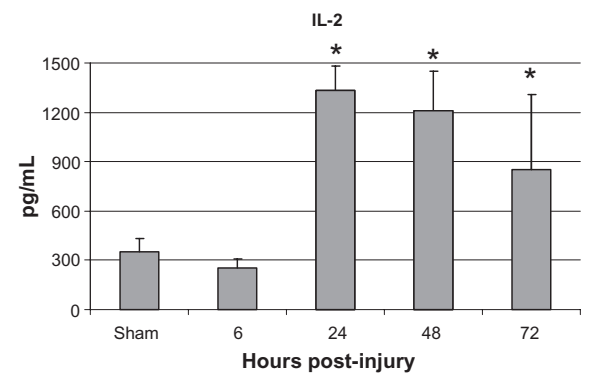

$\mathbf{F}$

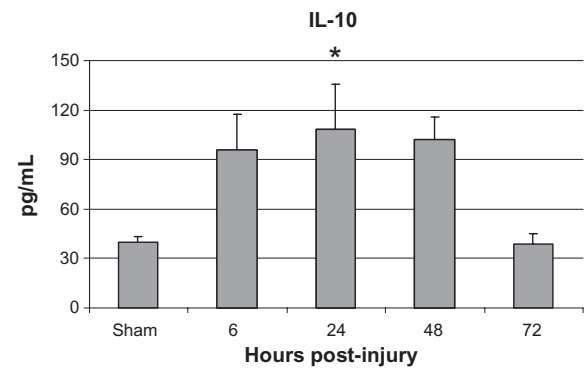

G

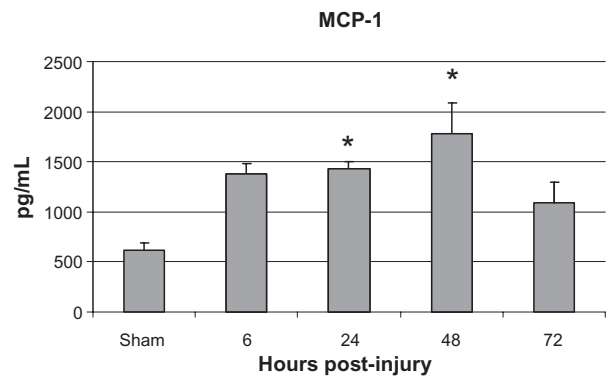

Figure 3 Serum levels of A) GRO/KC, B) IL-6, C) GM-CSF, D) IL-2, E) WBC, F) IL- I0, and G) MCP-I.

Notes: Data is shown as mean \pm SEM for the sham surgery group as well as the $6,24,48$, and 72 hours post-injury groups. $* P<0.05$ when compared with sham group, one-way ANOVA with Tukey's post-hoc test.

\section{Discussion}

The use of cytokines and other markers of inflammation as outcome prediction tools have the potential for clinical use in a variety of medical fields. During treatment of trauma, screening post-injury inflammatory biochemical markers is becoming increasingly investigated and has shown the potential for clinical use in medicine, including identification of optimal timing for traumatic wound closure, ${ }^{17}$ predicting mortality in patients after severe traumatic brain injury, ${ }^{18}$ and predicting the development of multiorgan failure. ${ }^{19}$ It is well established that inflammatory-associated lung injury represents a major source of mortality following serious orthopedic trauma. ${ }^{1,2}$ Assessment of inflammatory markers during heightened systemic inflammatory response, as well as inflammatory-related pulmonary injury, may assist in determining appropriate resuscitation and optimizing the timing of surgical intervention. 
A

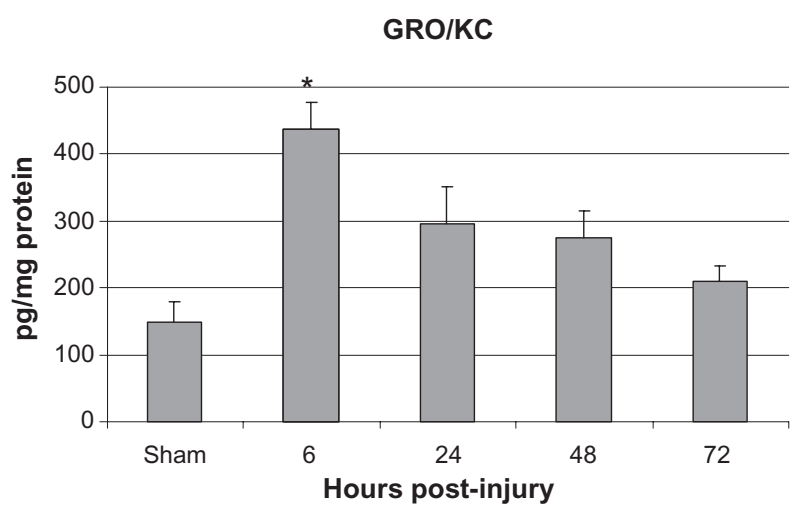

B

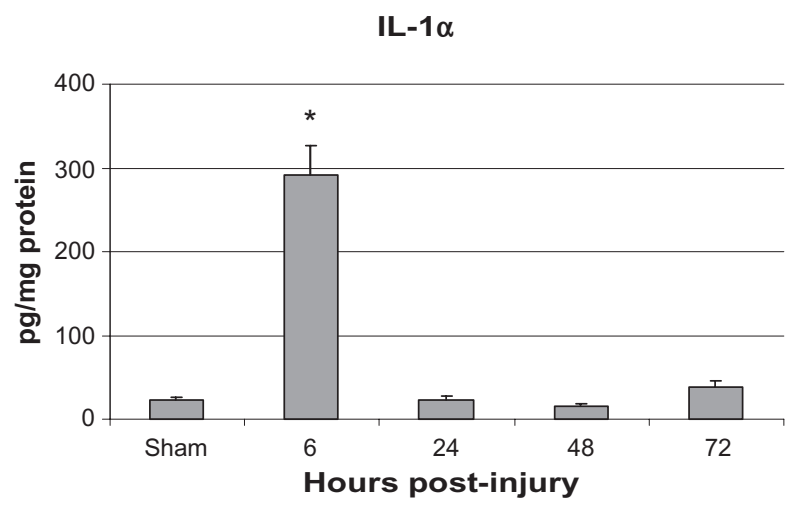

C

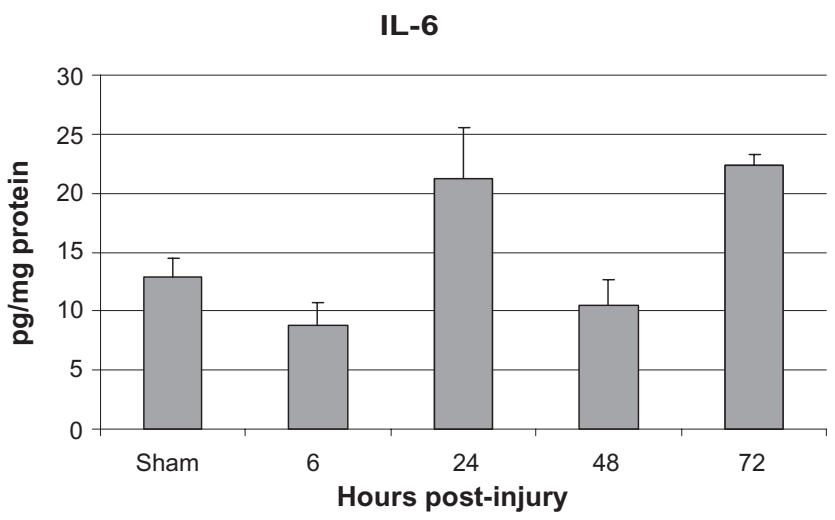

Figure 4 Lung levels of A) GRO/KC, B) IL-I $\alpha$, and C) IL-6 in pg/mg protein.

Notes: Data is shown as mean \pm SEM for the sham surgery group as well as the $6,24,48$, and 72 hours post-injury groups. $* P<0.05$, when compared with sham group, one-way ANOVA with Tukey's post-hoc test.

A clinical study report has shown the mortality rate of $30 \%$ following femoral shaft fractures. ${ }^{20}$ In rodent studies, previous investigations have shown that bilateral femur fracture with fixation results in inflammatory-associated injury to lung tissue. ${ }^{14,20}$ Our findings indicate that bilateral femur fracture with stabilization results in the significant elevation of a number of clinically important markers of inflammation in both serum and lung tissue. Significantly elevated serum levels of the human IL-8 homolog, $\mathrm{KC} /$ GRO (CINC-1), was the only marker that also demonstrated significantly increased post-injury levels in lung tissue. Both serum and lung levels of this cytokine were found to be significantly elevated at the same time point (6 hours) after injury. IL-6 levels in pulmonary tissue were associated with an increased trend, but did not reach significance.

Elevation of serum and lung inflammatory markers after bilateral femur fracture with stabilization also coincided with significant histological changes in lung tissue. Compared with controls, animals euthanized 6 hours after injury were found to have parenchymal and alveolar leukocyte infiltration, perivascular leukocyte infiltration, pulmonary edema and bone marrow emboli. Injured animals euthanized at 24 hours also demonstrated microscopic changes when compared with sham animals; however, these changes had dissipated substantially from those found at 6 hours post-injury. Increased serum and lung levels of KC/GRO (CINC-1) was reported to occur at the same time point in which the most significant changes in lung histology were observed ( 6 hours postinjury), although it is unclear if either the extremity or lung injury was the precipitous for this finding.

Murine growth-related oncogene (GRO) and chemokine CXCL1 (also termed KC) are members of same chemoattractant cytokine family as human IL-8, and demonstrate structural and functional homology to IL-8. ${ }^{21}$ Human IL-8 is a potent neutrophil chemoattractant and possesses neutrophilic activating properties resulting in the stimulation of phagocytosis, the generation of superoxide radicals, and the degranulation of cytoplasmic granules. ${ }^{22}$ In a pulmonary tissue injury model (lung reperfusion syndrome), elevation in IL-8 levels were associated with neutrophil infiltration 
A

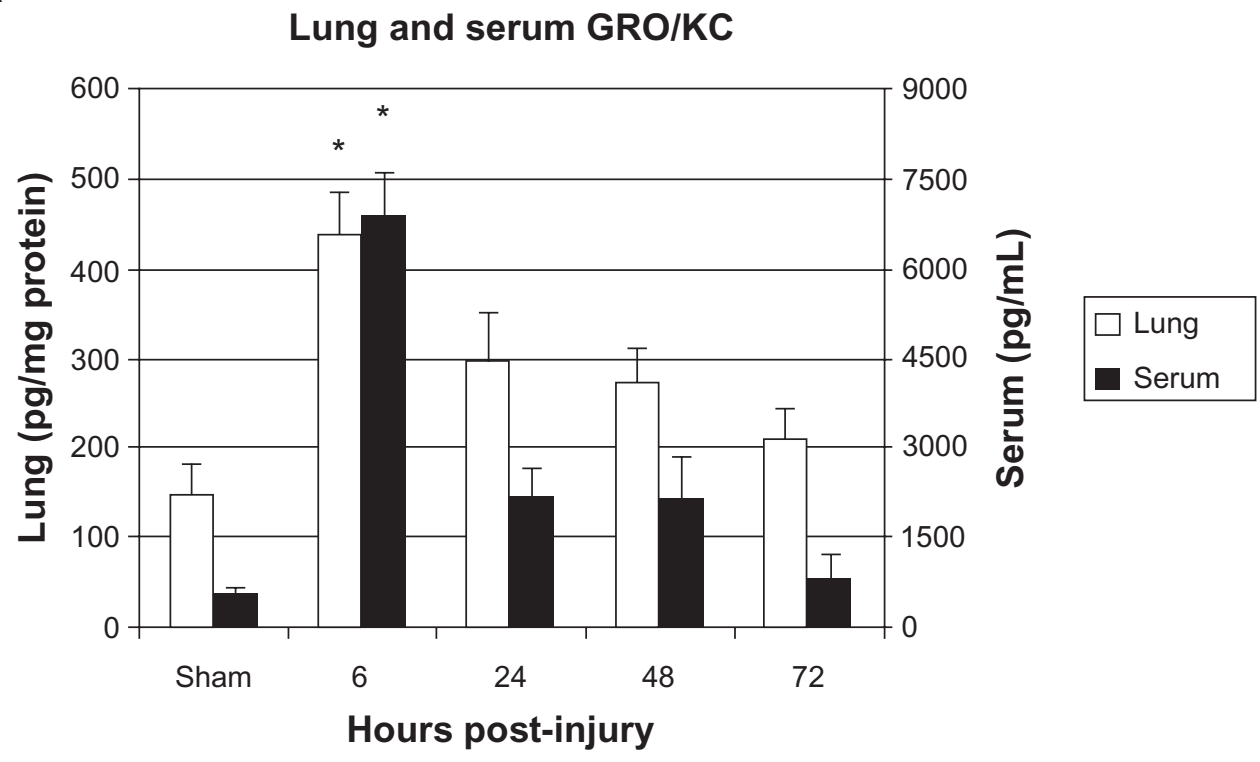

B

\section{Lung and serum IL-6}

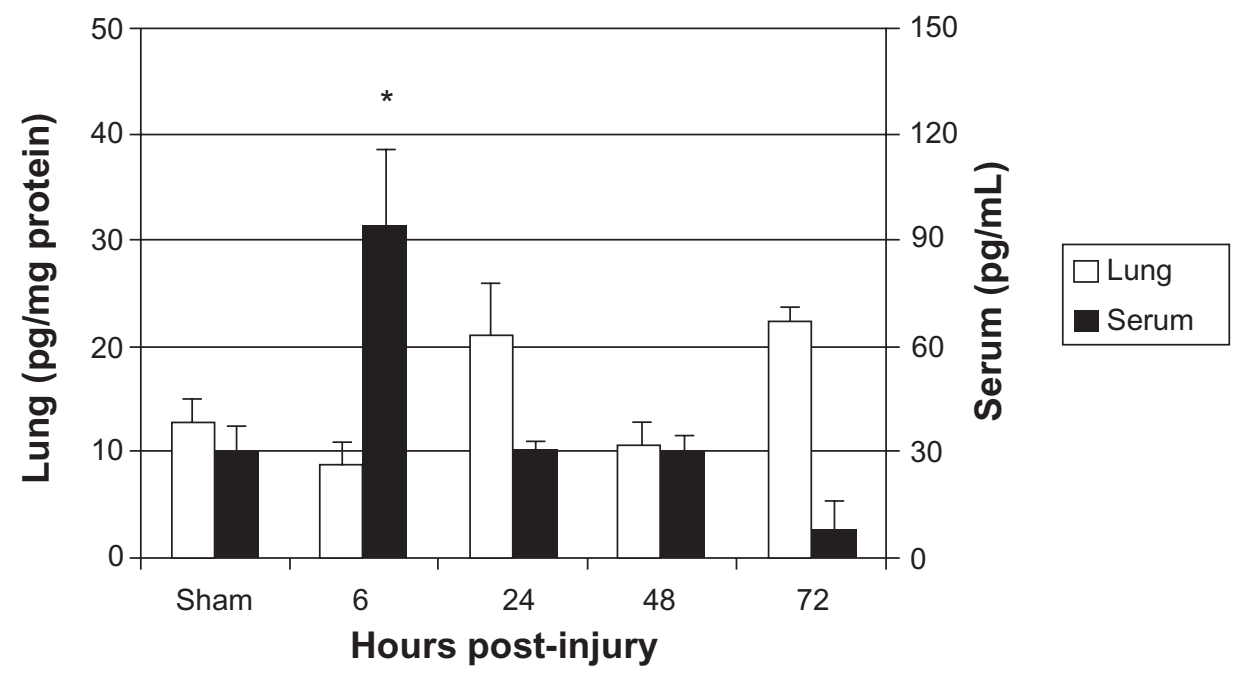

Figure 5 Serum and lung levels of A) GRO/KC and B) IL-6.

Notes: Data is shown as mean \pm SEM for the sham surgery group as well as the $6,24,48$, and 72 hours post-injury groups. $* P<0.05$, when compared with sham group, one-way ANOVA with Tukey's post-hoc test.

into lung tissue and increased levels of neutrophils in bronchoalveloar lavage fluid, suggesting that IL-8 is a major mediator involved in neutrophil recruitment and neutrophildependent tissue damage. ${ }^{23}$ In addition to the pulmonary injury, fracture fluid (hematoma combined with fracturegenerated cellular debris) released into circulation has been shown to contain significant levels of IL-8 and initiates the release of oxidative mediators (respiratory burst) within pulmonary tissue. ${ }^{24}$ Clinically, IL-8 levels have been shown to be elevated in traumatic deaths, ${ }^{7}$ but not in nontraumatic deaths, and serum levels at admission have been identified as the most important determinant of post-injury mortality in children following blunt trauma. ${ }^{8}$

Histological analysis in this study demonstrated pathologic changes in pulmonary tissue following bilateral femur fracture with fixation. These changes notably consisted of perivascular leukocyte infiltration (at 6 hours), possibly as a result of fracture elements lodging in pulmonary vessels, with accompanying vascular congestion and early pulmonary edema. As these bone elements are cleared, leukocytes migrate into peribronchiolar tissue, as seen in lung tissue samples at 24 hours post-injury. These findings 

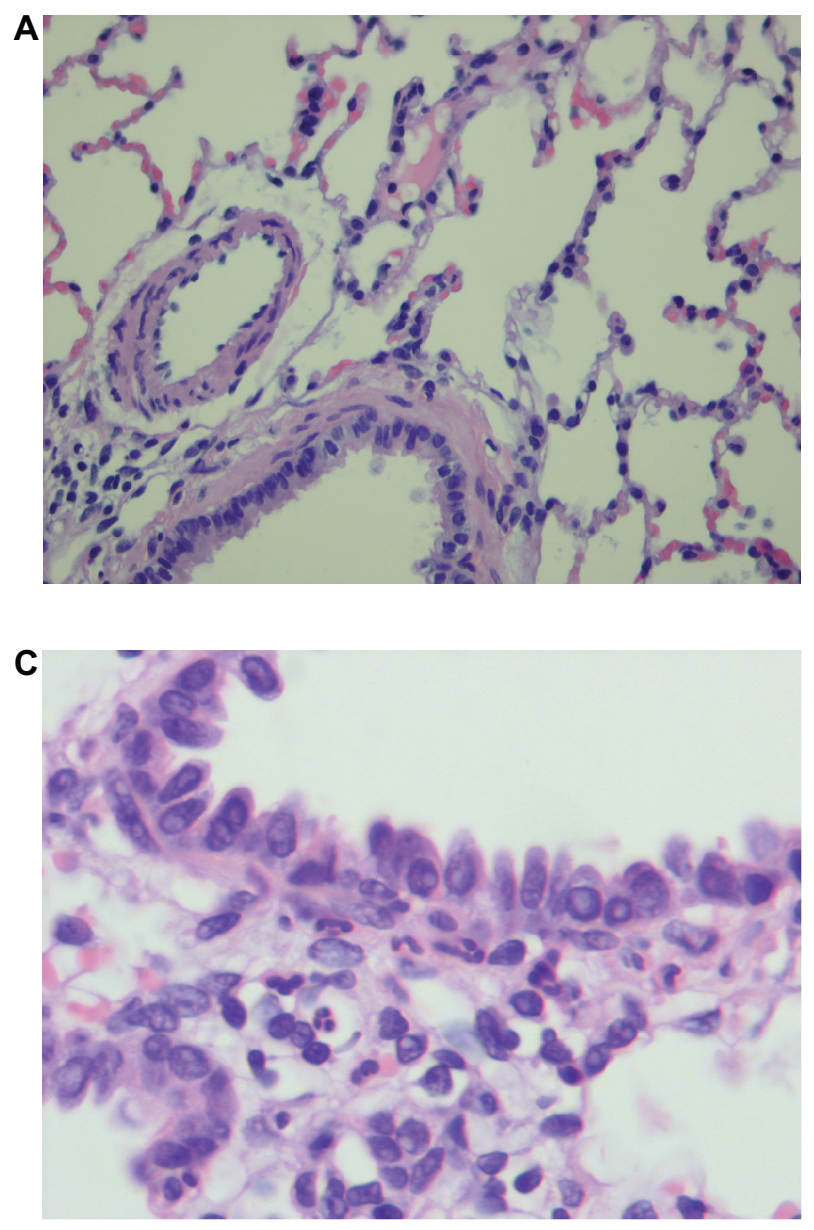

B
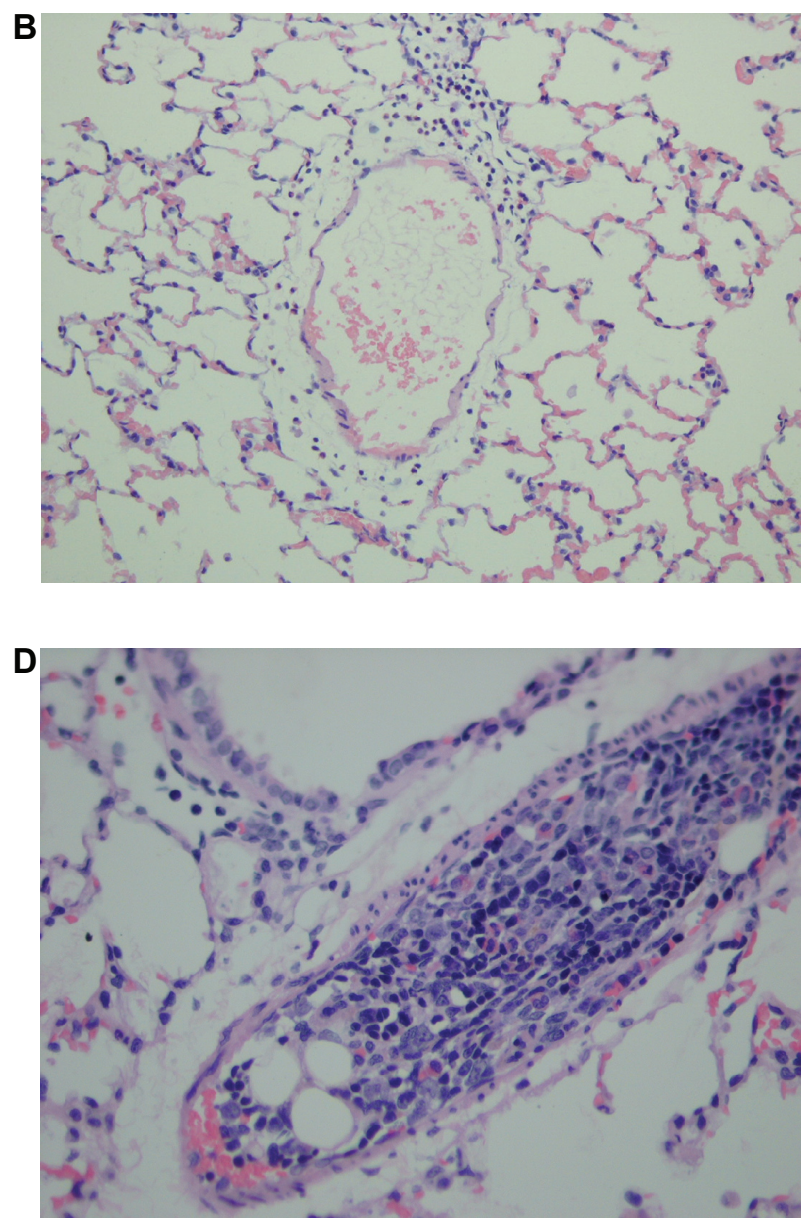

Figure 6 Histology slides of pulmonary tissue at 6 hours and 24 hours sham and post-injury. A) Normal alveolated lung parenchyma with normal bronchiolar vascular structures (400 $\times$ H\&E), B) Perivascular leukocyte infiltration at 6 hours after femur fracture, Grade II (200 $\times$ H\&E), C) Peribronchiolar leukocyte infiltration at 24 hours after femur fracture, Grade I ( $1000 \times \mathrm{H} \& \mathrm{E})$, and D) Bone marrow embolus filling vascular lumen (H\&E stain $400 \times)$.

correlate with results published by Yamamoto regarding the pathogenesis of pulmonary bone marrow embolism. ${ }^{25}$ In his study, Yamamoto examined pulmonary changes in rabbits during a 24-hour period after infusing $500 \mathrm{mg}$ of allogeneic bone marrow into the venous system. The author found that pulmonary leukostasis was highest at 5-10 hours after infusion, but by 24 hours had decreased considerably. In perivascular connective tissue, the author reported that edema and inflammatory cell infiltration may have occurred as a result of increased vascular permeability. Although this mechanism was not investigated further, mechanical injury to vascular endothelial cells as a result of bone marrow and fragment emboli could lead to increased vascular congestion, permeability, and the accumulation of leukocytes in the pulmonary vessels. Systemically and locally, pulmonary neutrophil infiltration has been linked to the cytokine IL-8. ${ }^{23,24}$ Although not formally investigated, heightened levels of IL- 8 seen in this study may be secondary to pulmonary vascular congestion and perivascular leukocyte infiltration (although the amount of marrow emboli was not quantified), which can be associated with long bone fracture or reperfusion.

In the current investigation, pathological alterations occurred in pulmonary tissue after bilateral femur fracture with fixation; however, the clinical significance of these specific changes cannot be determined. Previous studies examining pulmonary injury with an isolated fracture model have found marrow fat embolus to the lungs to be transient and not clinically significant. ${ }^{26}$ Conversely, when associated with thoracic trauma, fat embolus from long bone fracture has been shown to contribute to impaired pulmonary function. ${ }^{27}$ Additionally, although pulmonary histological changes resulting from orthopedic trauma may not necessarily be clinically important primarily, pulmonary injury potentially predisposes the patient to adverse outcomes from a second hit mechanism, including operative 
procedures. ${ }^{3,5}$ In this study, both serum and lung levels of the human IL-8 homolog, $\mathrm{KC} / \mathrm{GRO}$ (CINC-1), were shown to be significantly elevated at the same time point after orthopedic trauma that histological changes was identified in pulmonary tissue (6 hours post-injury). Levels of this marker returned to baseline as perivascular leukocyte infiltration cleared and lung changes dissipated. Little is known of the importance of isolating specifically elevated cytokine levels in pulmonary tissue when determining magnitude of lung inflammation response, other than these markers that are representative of an inflammatory process. Results from this study indicate that serum IL-8 levels (or in the murine model, GRO/KC) may be a useful inflammatory marker to evaluate for indications of pulmonary inflammation after orthopedic trauma.

There are several limitations to this study. To begin with, this study is based on the rodent model. The timing and magnitude of the inflammatory response following injury in rodents is likely different than that occurring in humans. However, used as a proof of concept model, this information adds to our understanding of the immunobiology of traumatic injury and gives us a platform to design clinical-based studies to better understand this important phenomenon in critically injured patients. Further, our injury model required femur fixation to occur at the same time as fracture, which clearly deviates from the typical clinical timeline of a trauma patient requiring operative fixation. This model was used primarily because pinning of fractures at time of injury was an IACUC requirement established to protect animal welfare (ability to eat and drink post-injury). In addition, this model has been established as an accepted closed femur fracture model within the literature. ${ }^{15}$ Although this model deviates from the typical clinical timeline of most trauma patients, we believe that our model still adequately represents the inflammatory response following bilateral long bone fracture secondary to the mechanism of fracture (blunt guillotine) with associated soft tissue injury. Lastly, the results of this study do not provide sufficient information to determine the clinical significance or outcome associated with elevated inflammatory markers or pulmonary histological changes following bilateral femur fracture with fixation. Although clinical studies are required, we believe that information gained from this study will eventually be applied to the clinical evaluation of the inflammatory status in polytrauma patients with long bone fractures.

In conclusion, results of this study indicate that bilateral femur fracture with stabilization in rats causes an increase in measureable serum markers of inflammation. Among the inflammatory markers screened, the rise in serum $\mathrm{KC} / \mathrm{GRO}$
(CINC-1), homolog to human IL-8, was found to correlate with elevation in levels in pulmonary tissue, both occurring at the earliest time point post-injury ( 6 hours). The rise in serum and lung levels of this inflammatory marker also correlated with histological changes in pulmonary tissue. These histological changes were consistent with inflammatory injury, including parenchymal and alveolar leukocyte infiltration, perivascular leukocyte infiltration, pulmonary edema, and bone marrow emboli. After 24 hours, serum and lung levels of $\mathrm{KC} / \mathrm{GRO}$ (CINC-1) returned to baseline with resolving histological changes. Thus, in this particular rat model, serum analysis of human IL-8, homolog to rat $\mathrm{KC} / \mathrm{GRO}$ (CINC-1), may provide an accessible marker with potential for evaluating inflammatory-associated lung changes following significant orthopedic injury. Ultimately, this marker may be useful as an adjunct to help guide clinical decisions regarding surgical timing.

\section{Notes}

This study was supported in part by the The National Institute of Health, National Institute on Alcohol Abuse and Alcoholism grant RO1 AA016138 to JJC.

This study was presented in poster format at the 2009 Mid-America Orthopaedic Association Meeting in Amelia Island, FL.

\section{Disclosure}

The authors report no conflicts of interest in this work.

\section{References}

1. Giannoudis PV. Current concepts of the inflammatory response after major trauma: an update. Injury. 2003;34:397-404.

2. Pape HC, Rixen D, Morley J, et al. Impact of the method of initial stabilization for femoral shaft fractures in patients with multiple injuries at risk for complications (borderline patients). Ann Surg. 2007;246 491-499.

3. Waydhas C, Nast-Kolb D, Trupka A. Posttraumatic inflammatory response, secondary operations and later multiple organ failure. J Trauma. 1996;40:624-631.

4. Visser T, Pillay J, Koenderman L, et al. Postinjury immune monitoring: can multiple organ failure be predicted? Curr Opin Crit Care. 2008;14 666-672.

5. Terminella L, Sharma G. Diagnostic studies in patients with acute respiratory distress syndrome. Semin Thorac Cardiovasc Surg. 2006;18: $2-7$.

6. Gebhard F, Pfetsch H, Steinbach G. Is interleukin 6 an early marker of injury severity following major trauma in humans? Arch Surg. 2000;135: 291-295.

7. Mimasaka S, Funayama M, Hashiyada M, et al. Significance of levels of IL-6 and IL-8 after trauma: a study of 11 cytokines post-mortem using multiplex immunoassay. Injury. 2007;38:1047-1051.

8. Ozturk H, Yagmur Y, Ozturk H. The prognostic importance of serum IL-1beta, IL-6, IL-8 and TNF-alpha levels compared to trauma scoring systems for early mortality in children with blunt trauma. Pediatr Surg Int. 2008;24:235-239. 
9. Giannoudis PV, Hildebrand F, Pape HC. Inflammatory serum markers in patients with multiple trauma. J Bone Joint Surg Br. 2004;86: 313-323.

10. Giannoudis PV, Smith MR, Evans RT, et al. Serum CRP and IL-6 levels after trauma: not predictive of septic complications. Acta Orthop Scand. 1998;69:184-188.

11. Osuchowski MF, Connett J, Welch K, Granger J, Remick DG. Stratification is the key: inflammatory biomarkers accurately direct immunomodulatory therapy in experimental sepsis. Crit Care Med. 2009;37:1567-1573.

12. Pape HC, van Griensven M, Rice J, et al. Major secondary surgery in blunt trauma patients and perioperative cytokine liberation: determination of the clinical relevance of biochemical markers. J Trauma. 2001; 50:989-1000.

13. Donnelly M, Condron C, Murray P, Bouchier-404 Hayes D. Modulation of the glycemic response using insulin attenuates the pulmonary response in an animal trauma model. J Trauma. 2007;63:351-357.

14. Timlin M, Condron C, Toomey D, et al. N-acetylcysteine attenuates lung injury in a rodent model of fracture. Acta Orthop Scand. 2004; 75:61-65.

15. Bonnarens F, Einhorn TA. Production of a standard closed fracture in laboratory animal bone. J Orthop Res. 1984;2:97-101.

16. Aytacoglu BN, Calikoglu M, Tamer L, et al. Alcohol-induced lung damage and increased oxidative stress. Respiration. 2006;73:100-104.

17. Forsberg JA, Elster EA, Andersen RC, et al. Correlation of procalcitonin and cytokine expression with dehiscence of wartime extremity wounds. J Bone Joint Surg Am. 2008;90:580-588.

18. Lo TY, Jones PA, Minns RA. Pediatric brain trauma outcome prediction using paired serum levels of inflammatory mediators and brain specific proteins. J Neurotrauma. 2009;26:1479-1487.
19. Maier B, Lefering R, Lehnert M, et al. Early versus late onset of multiple organ failure is associated with differing patterns of plasma cytokine biomarker expression and outcome after severe trauma. Shock. 2007; 28:668-674.

20. Copeland CE, Mitchell KA, Brumback RJ, Gens DR, Burgess AR. Mortality in patients with bilateral femoral fractures. J Orthop Trauma. 1998; 12:315-319

21. Himi T, Yoshioka I, Kataura A. Production and gene expression of IL-8-like cytokine GRO/CINC-1 in rat nasal mucosa. Acta Otolaryngol. 1997; 117:123-127.

22. Harada A, Sekido N, Akahoshi T, et al. Essential involvement of interleukin-8 (IL-8) in acute inflammation. J Leukoc Biol. 1994;56: 559-564.

23. Sekido N, Mukaida N, Harada A, et al. Prevention of lung reperfusion injury in rabbits by a monoclonal antibody against interleukin-8. Nature. 1993;365:654-657.

24. Hauser CJ, Desai N, Fekete Z, et al. Priming of neutrophil $[\mathrm{Ca} 2+] \mathrm{i}$ signaling and oxidative burst by human fracture fluids. JTrauma. 1999; 47:854-858.

25. Yamamoto M. Pathology of experimental pulmonary bone marrow embolism. Initial lesions of the rabbit lung after intravenous infusion of allogeneic bone marrow with special reference to its pathogenesis. Acta Pathol Jpn. 1985;35:45-69.

26. Schemitsch EH, Jain R, Turchin DC, et al. Pulmonary effects of fixation of a fracture with a plate compared with intramedullary nailing. A canine model of fat embolism and fracture fixation. $J$ Bone Joint Surg Am. 1997;79:984-996.

27. Elmaraghy AW, Aksenov S, Byrick RJ, et al. Pathophysiological effect of fat embolism in a canine model of pulmonary contusion. J Bone Joint Surg Am. 1999;81:1155-1164.
Journal of Inflammation Research

\section{Publish your work in this journal}

The Journal of Inflammation Research is an international, peer-reviewed open-access journal that welcomes laboratory and clinical findings on the molecular basis, cell biology and pharmacology of inflammation including original research, reviews, symposium reports, hypothesis formation and commentaries on: acute/chronic inflammation; mediators of inflamma-

\section{Dovepress}

tion; cellular processes; molecular mechanisms; pharmacology and novel anti-inflammatory drugs; clinical conditions involving inflammation. The manuscript management system is completely online and includes a very quick and fair peer-review system. Visit http://www.dovepress.com/ testimonials.php to read real quotes from published authors. 